\title{
A Novel Paclitaxel-Loaded Polymeric Micelle System with Favorable Biocompatibility and Superior Antitumor Activity
}

\author{
CAIYUN DENG ${ }^{1}$, CHANG XU $^{2}$, XIAOMIN ZHANG $^{2}$, JU YAO $^{2}$, YINGXIN ZHANG ${ }^{2}$, \\ BO $\mathrm{YU}^{2}$, ROBERT J. LEE $^{3}$ and CHENGJUN JIANG ${ }^{1}$ \\ ${ }^{1}$ Department of Chemical and Biological Engineering, \\ Zhejiang University of Science \& Technology, Hangzhou, Zhejiang, P.R. China; \\ ${ }^{2}$ Hangzhou Push-Kang Biotechnology Co., Ltd., Hangzhou, Zhejiang, P.R. China; \\ ${ }^{3}$ Division of Pharmaceutics and Pharmaceutical Chemistry, \\ College of Pharmacy, The Ohio State University, Columbus, OH, U.S.A.
}

\begin{abstract}
Background/Aim: Polymeric micelles are promising vehicles for paclitaxel delivery. Further improvement in the stability of the micelle formulation is desirable. Materials and Methods: Monomethoxy poly(ethylene glycol)-block-poly(D,Llactide)-9-fluorenylmethoxycarbonyl-L-phenylalanine (mPEGPDLLA-Phe(Fmoc)) was synthesized through a classical esterification reaction. Paclitaxel-loaded mPEG-PDLLAPhe(Fmoc) micelles (PTX-PheMs) were prepared by the selfassembly method. Composition, structure and physicochemical properties were characterized. Pharmacokinetics were evaluated in rats. Therapeutic effect was evaluated in tumorbearing mice. Safety profile was assessed by a hemolysis assay and an acute-toxicity study. Results: The average size of PTXPheMs was about $45 \mathrm{~nm}$. The hemolysis and acute-toxicity tests confirmed its biocompatibility and safety. The pharmacokinetics and therapeutic effect experiments demonstrated its long circulation property and superior antitumor effect. Conclusion: mPEG-PDLLA-Phe(Fmoc) micelle is a biocompatible and effective drug delivery system for hydrophobic drugs such as PTX.
\end{abstract}

Paclitaxel (PTX) is a mitotic inhibitor that presents high therapeutic efficacy against a wide range of solid tumors. The clinical advances of PTX have been limited due to its dose-limiting neurotoxic adverse effects and hypersensitivity caused by Cremophor EL (1). To overcome these limitations,

Correspondence to: Chengjun Jiang, Zhejiang University of Science \& Technology, Hangzhou, Zhejiang, P.R. China. Tel: +86 57185070383, e-mail: jcj312@163.com or Robert J. Lee, The Ohio State University, Columbus, OH, U.S.A. Tel: +1 6142924172, e-mail: lee.1339@osu.edu

Key Words: Paclitaxel, polymeric micelle, biocompatibility, antitumor.
Abraxane was developed and approved by the U.S. FDA in 2005 (2). Although Abraxane has shown advantages over Taxol, a significant decrease in white blood cells, red blood cells, and allergic reactions have been associated its use (3). Other delivery systems studied include polymer conjugates, liposomes, nanoparticles and micelles (4). Among these, polymeric micelles have gained considerable attention for their unique core/shell structure and high loading capacity. Genexol ${ }^{\circledR}$-PM is a polymeric micellar formulation in clinical trial in U.S.A. and approved in the Republic of Korea (5).

Although current PTX-polymeric micelle formulations significantly improve PTX solubility and decrease its toxicity, their therapeutic efficacy is comparable to Taxol (6). Indeed, it has been frequently observed that PTX encapsulated in polymeric micelles has similar pharmacokinetics to that of PTX free drug. This might be caused by destabilization of the micelles after i.v. injection, as well as by rapid PTX release from the micelles (7). Improving the stability of micelles preparation in vivo is critical in further improvement of this formulation.

Monomethoxy poly(ethylene glycol)-block-poly(D,Llactide) (mPEG-PDLLA) has been used to construct micelles, and has been used in Genexol ${ }^{\circledR}$-PM $(5,8)$. $\pi-\pi$ stacking is a physical interaction among molecules with aromatic groups that can drive supramolecular self-assembly (9-11. Introducing $\pi-\pi$ stacking will likely increase the interaction between anticancer drugs and the cores of polymeric micelles. Herein, mPEG-PDLLA was modified by conjugation to 9fluorenylmethoxy carbonyl-L-phenylalanine (Fmoc-L-Phe) to obtain end-capped Monomethoxy poly(ethylene glycol)block-poly(D,L-lactide)-9-fluorenylmethoxy carbonyl-Lphenylalanine (mPEG-PDLLA-Phe(Fmoc)). PTX-loaded mPEG-PDLLA-Phe micelles (PTX-PheMs) were prepared and compared with PTX-loaded mPEG-PDLLA (PTX-Ms). The physicochemical properties of PTX-PheMs were characterized. In vitro and in vivo activities were systematically 

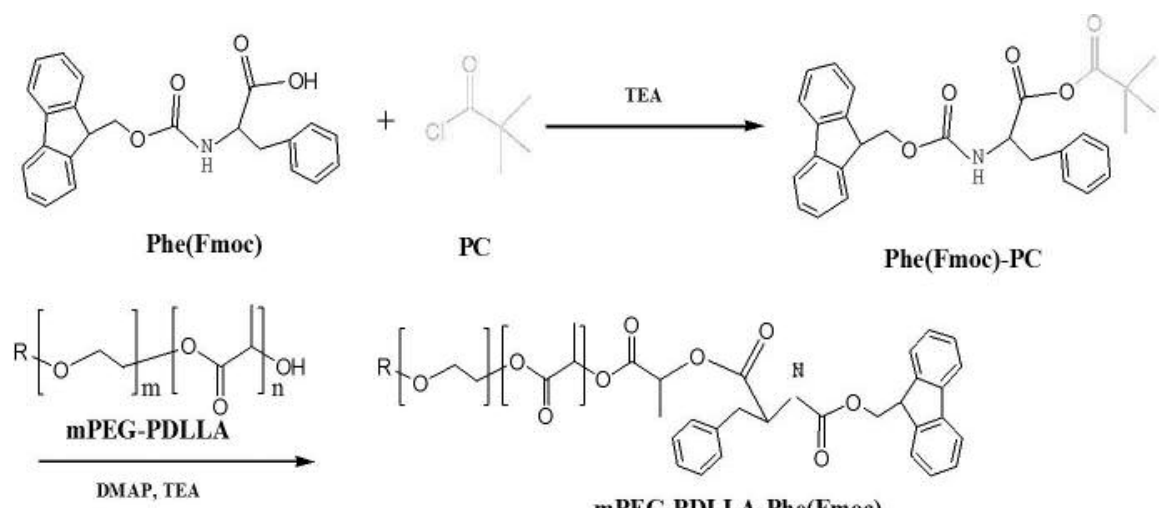

mPEG-PDLLA-Phe(Fmoc)

Figure 1. The synthetic routes of mPEG-PDLLA-Phe(Fmoc).

studied. Furthermore, hemolysis and acute toxicity were evaluated in order to assess the safety of PTX-PheMs.

\section{Materials and Methods}

Materials. PTX was purchased from Yew Biotechnology Co. (Jiangsu, P.R.China). BEL-7402 cells (human hepatocellular carcinoma cells) were obtained from Shanghai Institutes for Biological Sciences (Shanghai, P.R.China). Culture plates and dishes were purchased from Corning Inc. (New York, USA). Male Sprague-Dawley (SD) rats $(250 \pm 20 \mathrm{~g})$ and female BALB/c nude mice $(20 \pm 2 \mathrm{~g})$ were supplied by Animal Experiment Center of Zhejiang Academy of Medical Sciences (Hangzhou, P.R.China) and kept under SPF conditions. MPEG2000-PDLLA2000 was purchased from Advanced Polymer Materials Inc. Fmoc-L-Phe, pivaloyl chloride (PC), triethylamine (TEA) and 4-(dimethylamino)-pyridine (DMAP) were purchased from Aladdin Biochemical Technology Co., Ltd (Shanghai, P.R.China). Dry dichloromethane (DCM) was purchased from J\&K Scientific Ltd (Beijing, P.R.China). All other reagents and buffer solution components were of analytical grade.

Synthesis of mPEG-PDLLA-Phe (Fmoc). The conjugation of Fmoc-LPhe with the terminal hydroxyl group of mPEG-PDLLA was achieved using a mixed acid anhydride of Fmoc-L-Phe in the presence of DMAP (Figure 1) (12). In brief, Fmoc-L-Phe and TEA were dissolved in ethyl acetate and PC was added. The vessel was stirred at $0^{\circ} \mathrm{C}$ for $2 \mathrm{~h}$ then at room temperature (RT) for $1 \mathrm{~h}$. After filtration, the reaction solution was dried under vacuum. The obtained solid was dissolved in dry DCM and mixed with precooled dry DCM solution containing mPEG2000-PDLLA2000, TEA and DMAP. The reaction lasted for $2 \mathrm{~h}$ at $0^{\circ} \mathrm{C}$ then continued for $24 \mathrm{~h}$ at RT. The solvent was removed under vacuum. The crude product obtained was purified by silica gel column chromatography and collected liquid was dried in vacuum to give the final product mPEG2000PDLLA2000-Phe(Fmoc) (85.3\% yield). 1H NMR spectra was used for structure analysis recorded on a $500 \mathrm{MHz}$ Bruker nuclear magnetic resonance spectrometer (AVANCE III, Germany) at $25^{\circ} \mathrm{C}$. Critical micelle concentration (CMC) measurement of polymers. CMC of polymers were determined by pyrene as a florescent probe recorded on a Hitachi F-4600 spectrofluorometer in a spectral range of 350 to
$650 \mathrm{~nm}$. The fluorescence intensity ratio of $\mathrm{I}_{373} / \mathrm{I}_{384}$ was analyzed as a function of micelle concentration (13-14). The excitation wavelength of $335 \mathrm{~nm}$ with the scanning speed is $1,200 \mathrm{~nm} / \mathrm{min}$.

Micelle preparation. Micelles were easily prepared though film hydration method (15). In brief, antitumor drugs and mPEG2000PDLLA2000-Phe(Fmoc) were completely dissolved in acetone to obtain a clear solution. Acetone was removed on a rotary evaporator under reduced pressure to prepare drug-containing polymeric films. Then, the vessel was filled with water and rotated under normal pressure in a $37^{\circ} \mathrm{C}$ water bath to form micelle aqueous solution.

Size, zeta potential and morphological determination. Z-average diameter and zeta potential $(\xi)$ of nanoparticles were characterized by a Malvern Zetasizer (Nano-ZS, Malvern, Worcestershire, UK) at room temperature. Their morphology was observed through a JEM200C transmission electron microscope (TEM) from JEOL (Tokyo, Japan) at an acceleration voltage of $200 \mathrm{kV}$.

In vivo pharmacokinetic studies. Twelve male Sprague-Dawley rats $(250 \pm 20 \mathrm{~g})$ were randomly divided into three groups $(\mathrm{n}=4)$, and given a single $10 \mathrm{mg} / \mathrm{kg}$ dose of Taxol injection or PTX-contained micelles of mPEG2000-PDLLA2000-Phe(Fmoc). Then blood samples were collected at various times $(5,15,30 \mathrm{~min}, 1,2,4,8$, $12,24 \mathrm{~h}$ ) and treated according to previously described methods (16). The concentration of PTX was measured by RP-HPLC.

In vivo antitumor efficacy. In vivo tumor growth-inhibition was evaluated to assess the antitumor efficacy of PTX-PheMs. Specific procedures were as described in 17 . Tumor volume was calculated using formula: Volume=Length $\times \mathrm{Width}^{2} / 2$, and body weight was measured regularly as an indicator of antitumor efficacy.

Acute-toxicity experiment. To study the toxicity of the formulation, PTX-PheMs were injected as a single injection into five healthy male mice by tail vein at a concentration of $10 \mathrm{mg} / \mathrm{kg}$ (PTX equivalent). PBS was used as a negative control, and PTX injection was used as a positive control. Subsequently, the weight of the mice was measured for one week, and the blood samples were collected to determine routine blood and biochemical parameters. 

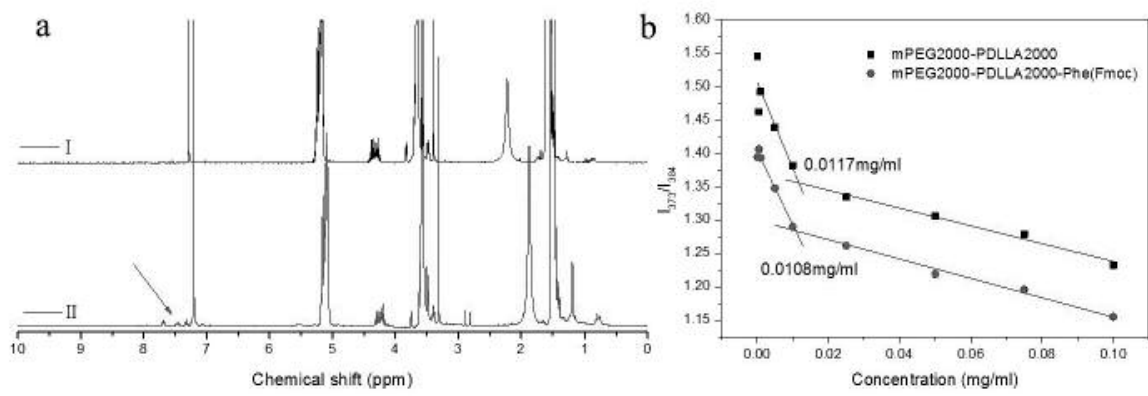

Figure 2. NMR spectrum and micelle formation of polymers. a) ${ }^{1} \mathrm{H}$ NMR spectrum of (I) mPEG2000-PDLLA2000 and (II) mPEG2000-PDLLA2000Phe (Fmoc) polymer in $\mathrm{CDCl}_{3}$. b) Fluorescence intensity ratio $\mathrm{I}_{373} / I_{384}$ (from fluorescence spectra) as a function of mPEG2000-PDLLA2000 and mPEG2000-PDLLA2000-Phe(Fmoc) copolymer concentrations at room temperature.

\section{Results}

Characterization of mPEG2000-PDLLA2000-Phe(Fmoc). The mPEG2000-PDLLA2000-Phe(Fmoc) copolymer was synthesized by esterification reaction between mPEG2000PDLLA2000 and acid anhydride of Fmoc-L-Phe. 1H NMR spectrum of the obtained copolymer is shown in Figure 2. Chemical shifts observed at $3.66 \mathrm{ppm}$ and at $5.23 \mathrm{ppm}$ belong to $-\mathrm{CH}_{2}$ protons in the PEG block and $-\mathrm{CH}$ protons in the PLA block, respectively. Compared with Figure $2 \mathrm{a}(\mathrm{I})$, clear resonances at $\delta 7.0 \sim 7.7 \mathrm{ppm}$ observed in Figure $2 \mathrm{a}$ (II) can be attributed to benzene ring or fused ring protons of the Fmoc-L-Phe residue, indicating the presence of Fmoc-L-Phe units in the copolymer chains. CMCs of mPEG2000-PDLLA2000 and mPEG2000-PDLLA2000Phe(Fmoc) were calculated to be 0.0117 and $0.0108 \mathrm{mg} / \mathrm{ml}$, respectively (Figure 2b). It indicated that Fmoc-L-Phe was incorporated in the polymer chain and enhanced its hydrophobicity. However, the conjugate remained soluble since Fmoc-L-Phe occupied a small proportion in the whole polymer chain.

Characterization of nanoparticles. PTX-Ms and PTX-PheMs were prepared though film hydration method and their particle size and zeta potential are summarized in Table I. Zaverage size of PTX-Ms and PTX-PheMs are $31.79 \mathrm{~nm}$ and $45.16 \mathrm{~nm}$, respectively. Meanwhile, both had a narrow distribution. The particle size distribution and TEM images are shown in Figure 3 and demonstrated low polydispersity and homogeneous size of particles.

In vitro hemolysis. Cremophor EL and ethanol, which are used in Taxol injection, can cause hemolysis. However, PTX-PheMs should overcome this problem. To confirm this, an in vitro hemolysis test was performed and the results are shown in Figure 4. They clearly show that hemolysis was not observed until the concentration of PTX-PheMs reaches
Table I. Mean $\pm S D$ size and zeta potential of PTX-Ms and PTX-PheMs $(n=3)$.

\begin{tabular}{lccc}
\hline Formulation & Size $(\mathrm{nm})$ & PDI & Zeta potential $(\mathrm{mV})$ \\
\hline PTX-Ms & $31.79 \pm 1.56$ & $0.171 \pm 0.042$ & $-10.86 \pm 0.85$ \\
PTX-PheMs & $45.16 \pm 3.22$ & $0.112 \pm 0.017$ & $-7.17 \pm 0.33$ \\
\hline
\end{tabular}

$500 \mu \mathrm{g} / \mathrm{ml}$. The results confirm that PTX-PheMs is safer and more biocompatible than Taxol.

Pharmacokinetics of PTX-PheMs. Compared with Taxol, PTXPheMs maintained a similar plasma concentration trend within the first $4 \mathrm{~h}$ (Figure 5a). But, after that the plasma concentration of PTX released from PTX-PheMs was much higher than that of Taxol. The formula of PTX-PheMs can maintain a higher plasma concentration in the body until $24 \mathrm{~h}$, which indicates improved systemic circulation. The area under the curve (AUC) for PTX-PheMs was significantly higher.

In vivo antitumor efficacy. Further, the anti-tumor efficacy of the formula was evaluated by using nude mice bearing Bel-7402 tumor. The results (Figure 5b) showed that similar antitumor efficacies were observed between PTX-PheMs and Taxol injection based on tumor volume changes within 18 days of beginning. After that, the tumor volume of the Taxol formula was gradually increased, while that of the PTXPheMs formula was slowly reduced to a constant level. The results indicated that the PTX-PheMs induced efficient and extensive intratumoral apoptosis. The body weight changes (Figure 5c) were monitored during the therapeutic study, demonstrating safety of PTX-PheMs formula.

Acute toxicity experiment. The biochemical analyses of mice showed that the PTX-PheMs group had no impact on any 

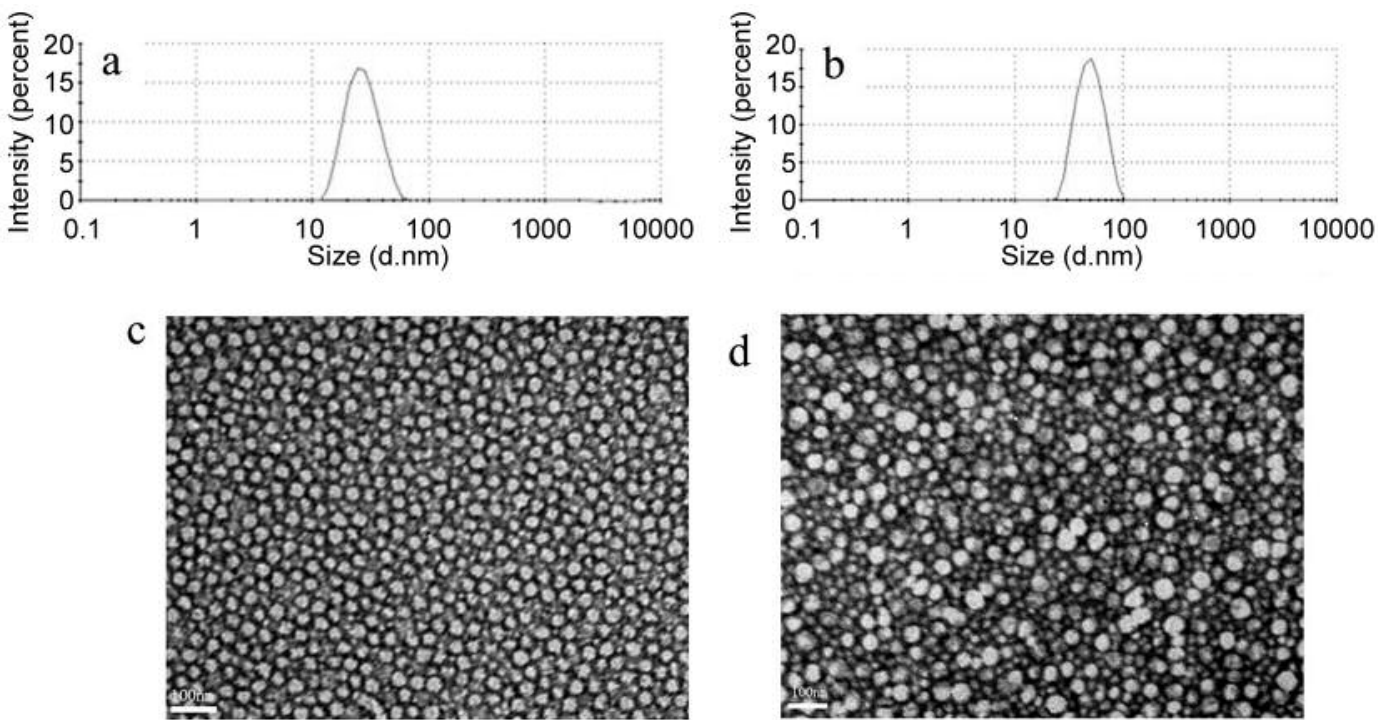

Figure 3. Particle size distribution and TEM images of PTX-Ms (a.c) and PTX-PheMs (b.d).

a

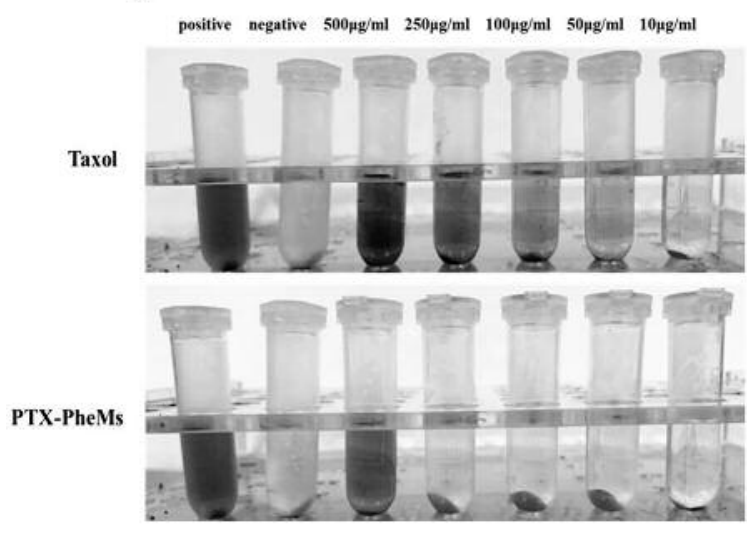

b

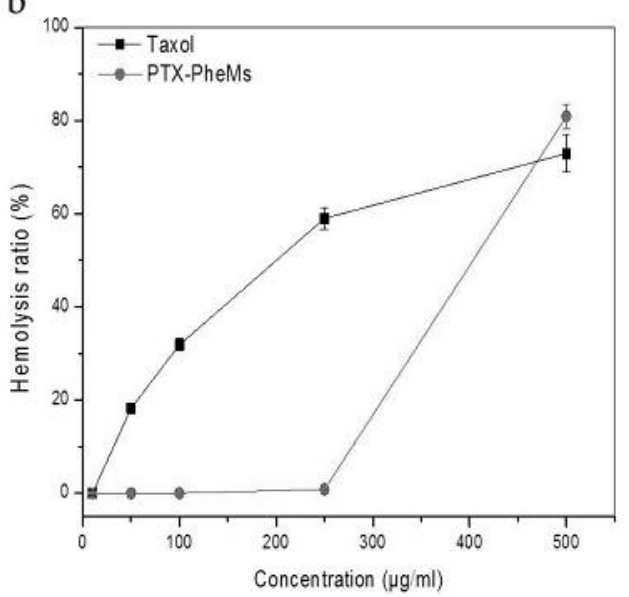

Figure 4. In vitro hemolysis. a) Image of hemolysis resulting from PTX-PheMs micelles and Taxol after centrifuging. b) The percentage of hemolysis induced by PTX-PheMs and Taxol (data are mean $\pm S D, n=3$ ).

indexes measured which indicated no hepatotoxicity (Figure 6). As shown in Table II, the results of routine blood tests of mice indicated that the value of mice was almost within normal range both for Taxol and PTX-PheMs groups.

\section{Discussion}

Ethanol and Cremophor EL used in Taxol, the clinical formulation of PTX injection, can cause hemolysis, allergies and other adverse reactions. Studies have shown that Taxol is metabolized faster in vivo (18-20). In order to overcome these limitation, we developed a new formulation for delivery of PTX. The water solubility of PTX was improved by selfassembly of amphiphilic block polymers of mPEG2000PDLLA2000-Phe(Fmoc) and PTX. In vivo pharmacokinetic studies showed that PTX-PheMs formula can keep plasma concentration at a high level and have a higher AUC value than Taxol. The results demonstrated that PTX of PTX-PheMs formula was slowly released, achieving long circulation of drug in vivo to a certain extent. This might be due to the structure of the terminal benzene ring of polymer, which interacts with the benzene ring of PTX through the $\pi-\pi$ interaction. In vivo 

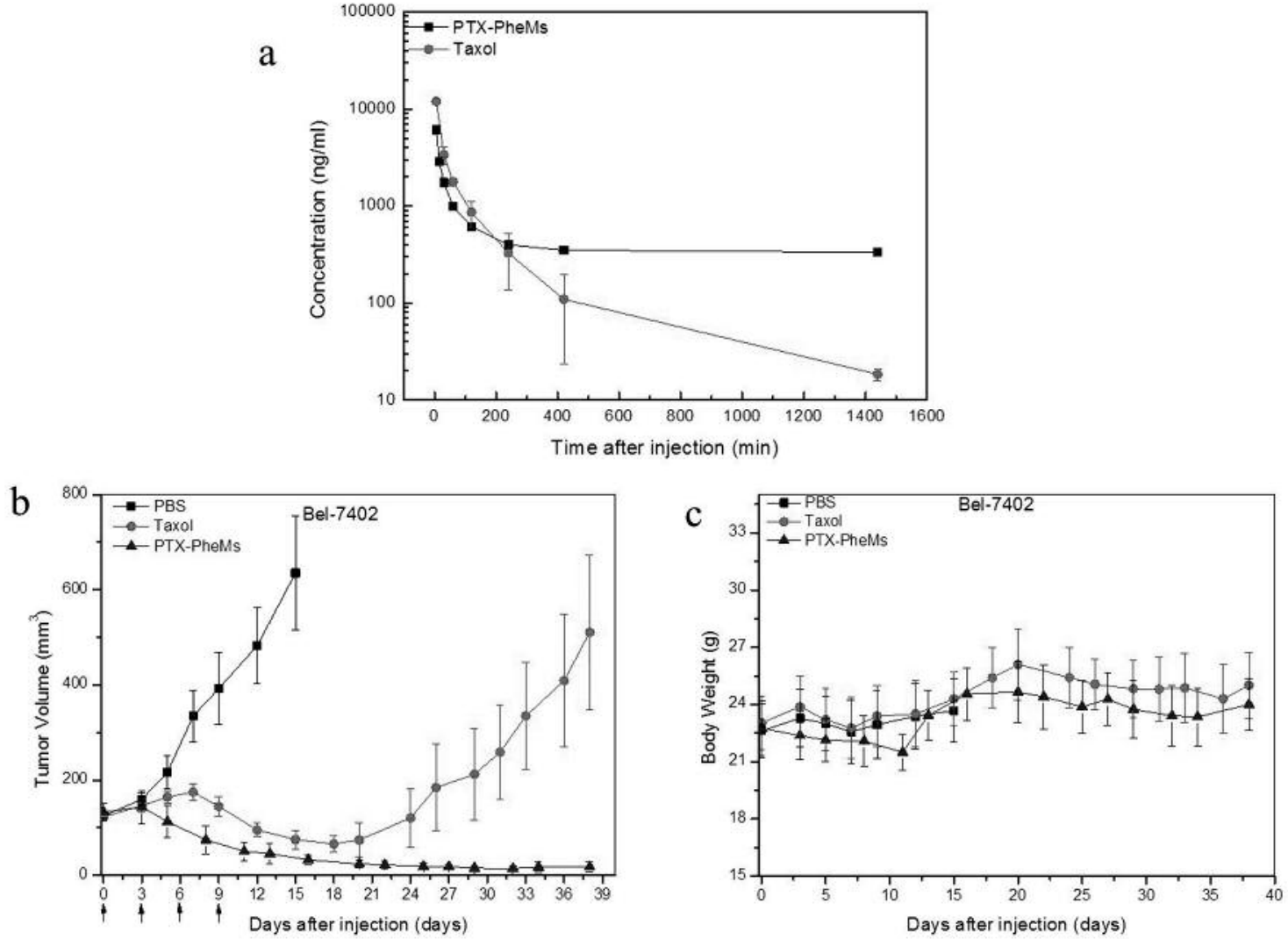

Figure 5. Pharmacokinetics and tumor growth inhibition. a) Pharmacokinetics of PTX-PheMs and Taxol (n=5). b) In vivo antitumor activity in an Bel-7402 xenograft mouse model treated with PTX-PheMs and Taxol via intravenous injection. $c$ ) Changes in body weight after treatment. Mice were treated with PBS, Taxol $(10 \mathrm{mg} / \mathrm{kg}$ ) and PTX-PheMs (at an PTX equivalent dose of $10 \mathrm{mg} / \mathrm{kg}$ ) at four successive times indicated with arrows $(n=6)$. The data are expressed as the means $\pm s . d . ; * p<0.05, * * p<0.01$.

antitumor efficacy experiment on BALB/c nude mice bearing Bel-7402 tumor showed that PTX-PheMs had more effectively inhibited the growth of the tumor compared to the PTX injection. Furthermore, in vitro hemolysis study demonstrated that PTX-PheMs could prevent hemolysis below the concentration of $500 \mu \mathrm{g} / \mathrm{ml}$. However, Taxol caused hemolysis as it contained Cremophor EL. Acute toxicity experiment showed no hepatotoxicity compared with PBS group and Taxol group. In summary, PTX-PheMs have superior pharmacodynamic behavior, antitumor efficacy and safety.

\section{Conclusion}

An amphiphilic block polymer terminal modified with a fused ring, mPEG2000-PDLLA2000-Phe(Fmoc), was synthesized. Micelles were prepared though self-assembly of mPEG2000PDLLA2000-Phe(Fmoc) and PTX. In vivo pharmacokinetic study showed a long circulation property of PTX-PheMs which can maintain a higher plasma concentration and AUC value than Taxol. In vitro hemolysis test showed the safety of PTX-PheMs.
In addition, in vivo antitumor efficacy data demonstrated excellent therapeutic efficacy. These results indicate that PTXPheMs are excellent carriers for poorly soluble PTX and has potential applications in the field of drug delivery.

\section{Acknowledgements}

This study was supported by the Zhejiang Provincial Natural Science Foundation, project No. LY15B60008.

\section{References}

1 Kim SC, Kim DW, Shim YH, Bang JS, Oh HS, Kim SW and Seo MH: In vivo evaluation of polymeric micellar paclitaxel formulation: toxicity and efficacy. J Control Release 72: 191202, 2001.

2 Sparreboom A, Scripture CD, Trieu V, Williams PJ, De T, Yang A, Beals B, Figg WD, Hawkins M and Desai N: Comparative preclinical and clinical pharmacokinetics of a cremophor-free, nanoparticle albumin-bound paclitaxel (ABI-007) and paclitaxel formulated in cremophor (Taxol). Clin Cancer Res 11: 41364143, 2005. 


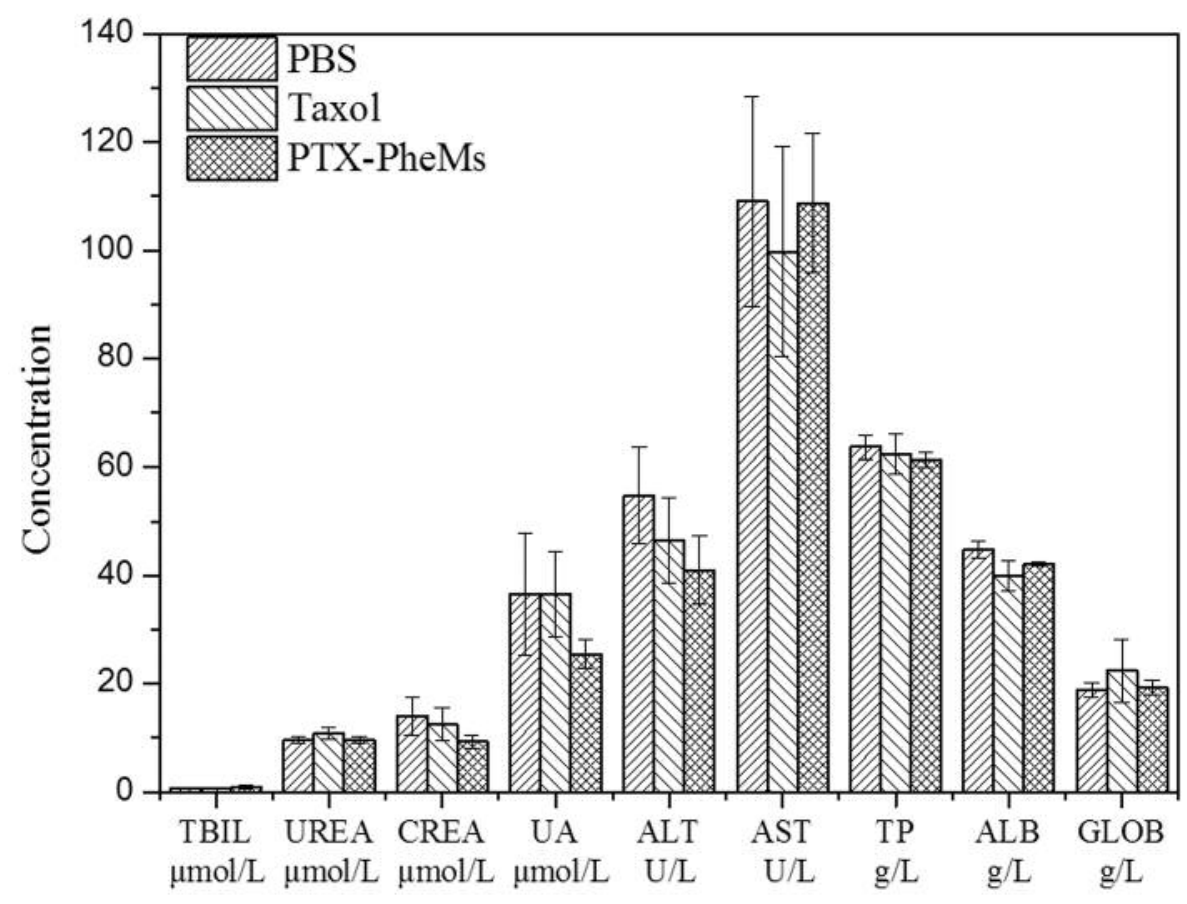

Figure 6. Blood biochemical analyses. T-BIL: Total bilirubin ( $\mu$ mol/L); UREA: carbamide (mmol/L); CREA: creatinine ( $\mu$ mol/L); UA: uric acid ( $\mu \mathrm{mol} / \mathrm{L}) ; A L T:$ alanine aminotransferase $(U / L) ; A S T:$ aspartate aminotransferase $(U / L) ; T P:$ total protein $(\mathrm{g} / L) ; A L B:$ albumin $(\mathrm{g} / L) ; G L O B$ : globulin $(g / L)$ (data are mean $\pm S D, n=5)$.

Table II. Results of routine blood test of mice (n=5), after treatment with PBS, Taxol and PTX-PheMs, respectively.

\begin{tabular}{|c|c|c|c|c|c|}
\hline Parameter & Unit & Reference & PBS & Taxol & PTX-PheMs \\
\hline WBC & $10^{9} / \mathrm{L}$ & 4-12 & $6.10 \pm 1.22$ & $4.60 \pm 0.50$ & $4.98 \pm 0.84$ \\
\hline $\mathrm{NE}$ & $10^{9} / \mathrm{L}$ & $0.7-4.0$ & $1.08 \pm 0.25$ & $0.90 \pm 0.64$ & $0.50 \pm 0.18$ \\
\hline LY & $10^{9} / \mathrm{L}$ & $3-8.5$ & $4.83 \pm 1.10$ & $3.55 \pm 0.61$ & $4.45 \pm 0.87$ \\
\hline MO & $10^{9} / \mathrm{L}$ & $0-1.3$ & $0.10 \pm 0.00$ & $0.15 \pm 0.10$ & $0.03 \pm 0.05$ \\
\hline EO & $10^{9} / \mathrm{L}$ & $0-0.5$ & $0.10 \pm 0.12$ & $0.00 \pm 0.00$ & $0.00 \pm 0.00$ \\
\hline BA & $10^{9} / \mathrm{L}$ & $0-0.1$ & $0.00 \pm 0.00$ & $0.00 \pm 0.00$ & $0.00 \pm 0.00$ \\
\hline NE\% & $\%$ & $12-44$ & $17.68 \pm 2.88$ & $19.48 \pm 12.70$ & $10.50 \pm 3.93$ \\
\hline LY\% & $\%$ & $54-85$ & $78.75 \pm 3.58$ & $77.23 \pm 15.09$ & $88.55 \pm 3.73$ \\
\hline $\mathrm{MO} \%$ & $\%$ & $0-15$ & $1.58 \pm 0.46$ & $3.13 \pm 2.55$ & $0.73 \pm 0.22$ \\
\hline $\mathrm{EO} \%$ & $\%$ & $0-5$ & $1.98 \pm 2.33$ & $0.15 \pm 0.17$ & $0.23 \pm 0.25$ \\
\hline BA\% & $\%$ & 0-1 & $0.03 \pm 0.05$ & $0.03 \pm 0.05$ & $0.00 \pm 0.00$ \\
\hline $\mathrm{RBC}$ & $10^{12} / \mathrm{L}$ & $7.7-12.5$ & $7.81 \pm 0.39$ & $7.66 \pm 0.55$ & $8.25 \pm 0.32$ \\
\hline HGB & $\mathrm{g} / \mathrm{L}$ & $120-200$ & $140.50 \pm 5.00$ & $141.50 \pm 9.26$ & $146.50 \pm 8.81$ \\
\hline HCT & $\mathrm{L} / \mathrm{L}$ & $25-56$ & $40.30 \pm 2.25$ & $38.55 \pm 2.00$ & $41.08 \pm 1.41$ \\
\hline $\mathrm{MCV}$ & $\mathrm{fL}$ & $40-48$ & $51.58 \pm 0.35$ & $50.38 \pm 1.33$ & $49.80 \pm 0.80$ \\
\hline $\mathrm{MCH}$ & pg & $20-32$ & $18.03 \pm 0.80$ & $18.48 \pm 0.41$ & $17.75 \pm 0.44$ \\
\hline $\mathrm{MCHC}$ & $\mathrm{g} / \mathrm{L}$ & $300-370$ & $349.00 \pm 17.34$ & $366.75 \pm 5.74$ & $356.25 \pm 10.08$ \\
\hline RDW & $\%$ & $11.2-16$ & $13.98 \pm 0.31$ & $14.18 \pm 0.57$ & $14.78 \pm 0.41$ \\
\hline PLT & $10^{9} / \mathrm{L}$ & $100-900$ & $520.50 \pm 79.38$ & $547.75 \pm 99.31$ & $520.75 \pm 48.82$ \\
\hline PCT & $\mathrm{L} / \mathrm{L}$ & $0.15-0.3$ & $0.33 \pm 0.06$ & $0.36 \pm 0.08$ & $0.33 \pm 0.04$ \\
\hline MPV & $\mathrm{fL}$ & 5-10 & $6.28 \pm 0.21$ & $6.45 \pm 0.33$ & $6.30 \pm 0.22$ \\
\hline PDW & FI & $9-17$ & $12.08 \pm 0.56$ & $12.35 \pm 0.71$ & $12.00 \pm 0.58$ \\
\hline
\end{tabular}

WBC: White blood cells; NE: neutrophil granulocytes; LY: lymphocytes; MO: monocytes; EO: eosinophilic granulocytes; BA: basophilic granulocytes; RBC: red blood cells; HGB: hemoglobin; HCT: hematocrit; MCV: mean corpuscular volume; MCH: mean corpuscular hemoglobin; MCHC: mean corpuscular hemoglobin concentration; RDW: red blood cell distribution width; PLT: blood platelet; PCT: plateletcrit; MPV: mean platelet volume; PDW: platelet distribution width. 
3 Gradishar WJ, Tjulandin S, Davidson N, Shaw H, Desai N, Bhar $\mathrm{P}$, Hawkins $\mathrm{M}$ and O'Shaughnessy $\mathrm{J}$ : Phase III trial of nanoparticle albumin-bound paclitaxel compared with polyethylated castor oil-based paclitaxel in women with breast cancer. J Clin Oncol 23: 7794-7803, 2005.

4 Mikhail AS and Allen C: Poly(ethylene glycol)-b-poly( $\varepsilon$ caprolactone) micelles containing chemically conjugated and physically entrapped docetaxel: synthesis, characterization, and the influence of the drug on micelle morphology. Biomacromolecules 11: 1273-1280, 2010.

5 Kim TY, Kim DW, Chung JY, Shin SG, Kim SC, Heo DS, Kim NK and Bang YJ: Phase I and pharmacokinetic study of genexol-PM, a cremophor-free, polymeric micelle-formulated paclitaxel, in patients with advanced malignancies. Clin Cancer Res 10: 3708-3716, 2004.

6 Letchford K and Burt HM: Copolymer micelles and nanospheres with different in vitro stability demonstrate similar paclitaxel pharmacokinetics. Mol Pharmaceut 9: 248-260, 2012.

7 Letchford K, Liggins R, Wasan KM and Burt H: In vitro human plasma distribution of nanoparticulate paclitaxel is dependent on the physicochemical properties of poly(ethylene glycol)-blockpoly(caprolactone) nanoparticles. Eur J Pharm Biopharm 71: 196-206, 2009.

8 Li Y, Xiao K, Luo J, Xiao W, Lee JS, Gonik AM, Kato J, Dong TA and Lam KS: Well-defined, reversible disulfide cross-linked micelles for on-demand paclitaxel delivery. Biomaterials 32: 6633-6645, 2011.

9 Song B, Wang Z, Chen S, Zhang X, Fu Y, Smet M and Dehaen $\mathrm{W}$ : The introduction of $\pi-\pi$ stacking moieties for fabricating stable micellar structure: formation and dynamics of disklike micelles. Angew Chem Int Edit 117: 4809-4813, 2005.

10 Janiak C: ChemInform Abstract: A Critical account on $\pi-\pi$ stacking in metal complexes with aromatic nitrogen-containing Ligands. J Cheminformatics 32: 3885-3896, 2000.

11 Yang X, Grailer JJ, Pilla S, Steeber DA and Gong S: Tumortargeting, pH-responsive, and stable unimolecular micelles as drug nanocarriers for targeted cancer therapy. Bioconjugate Chem 21: 496-504, 2010.

12 Fan Y, Chen G, Tanaka J and Tateishi T:L-Phe end-capped poly (L-lactide) as macroinitiator for the synthesis of poly (L-lactide)b-poly (L-lysine) block copolymer. Biomacromolecules 6: 30513056, 2005.
13 Wilhelm M, Zhao CL, Wang Y, Xu R and Winnik: Poly (styreneethylene oxide) block copolymer micelle formation in water: a fluorescence probe study. Macromolecules 24: 1033-1040, 1991.

14 Qiu LY, Wang RJ, Zheng C, Jin Y and Jin LQ: $\beta$-cyclodextrincentered star-shaped amphiphilic polymers for doxorubicin delivery. Nanomedicine 5: 193-208, 2010.

15 Lee SW, Yun MH, Jeong SW, In CH, Kim JY, Seo MH, Pai CM and Kim SO: Development of docetaxel-loaded intravenous formulation, Nanoxel-PM ${ }^{\mathrm{TM}}$ using polymer-based delivery system. J Control Release 155: 262-271, 2011.

16 Jiang CJ, Wang HX, Zhang XM, Sun ZB, Wang F, Cheng J, Xie HY, Yu B and Zhou L: Deoxycholic acid-modified chitooligosaccharide/mPEG-PDLLA mixed micelles loaded with paclitaxel for enhanced antitumor efficacy. Int J Pharmaceut 475: 60-68, 2014.

17 Fang T, Dong YH, Zhang XM, Xie K, Lin L and Wang HX: Integrating a novel SN38 prodrug into the PEGylated liposomal system as a robust platform for efficient cancer therapy in solid tumors. Int J Pharmaceut 512: 39-48, 2016.

18 Wilhelm C, Billotey C, Roger J, Pons JN, Bacri JC and Gazeau F: Intracellular uptake of anionic superparamagnetic nanoparticles as a function of their surface coating. Biomaterials 24: 1001-1011, 2003.

19 Zhao LY and Feng SS: Enhanced oral bioavailability of paclitaxel formulated in vitamin E-TPGS emulsified nanoparticles of biodegradable polymers: in vitro and in vivo studies. Journal of pharmaceutical sciences J Pharm SCI-US 99: 3552-3560, 2010.

20 Li C, Newman RA, Wu QP, Ke S, Chen W, Hutto T, Kan ZX, Brannan MD, Charnsangavej $C$ and Wallace S: Biodistribution of paclitaxel and poly (L-glutamic acid)-paclitaxel conjugate in mice with ovarian OCa- 1 tumor. Cance Chemoth Pharm 46: 416-422, 2000. 